\title{
Quantitative Analysis of the Fault-Tolerance of Pragmatic General Multicast (PGM) and Elastic Reliable Multicast (ERM) Protocols
}

\author{
Okonkwo O. Raphael Okonkwo ${ }^{1}$, Akpojaro Jackson ${ }^{2}$ and Anthony Achuenu ${ }^{3}$ \\ ${ }^{1}$ Department of Computer Science, Nnamdi Azikwe University, Awka, Anambra State, Nigeria \\ ${ }^{2}$ Department of Mathematical and Physical Sciences, College of Basic and Applied Sciences, Samuel \\ Adegboyega University, Ogwa, Edo State, Nigeria \\ ${ }^{3}$ Department of Computer Science, Auchi Polytechnic, Auchi, Edo State, Nigeria \\ jakpojaro@yahoo.com; tonynayou2003@yahoo.com
}

\begin{abstract}
Multicast communication protocols are not immune from failures as a result of packets being dropped due to a broken link or time out processes. Therefore, it is essential to understand how these failures can affect the overall performance of multicast protocols over the Internet. This paper compares the faulttolerance effect of two reliable multicast protocols: pragmatic general multicast (PGM) and elastic reliable multicast (ERM) in a situation where a multicast-aware node fails and the sub-nodes will have to request a repaired packet. A simulation model is developed in such a way that faults are randomly created on nodes and link for a specified period of time and the fault-tolerance effect on the two multicast protocols is analyzed. The model developed for this paper repeats the simulation for different network size, the results obtained show that the ERM protocol is better than the PGM as the size of the network increases. This finding is key while considering the improvement (or upgrade) of existing multicast protocols. The result is also significant at the early stage of designing new multicast protocols as it provides useful information in allocating scarce resource that can be appropriated to improve other infrastructure in the network.
\end{abstract}

Keywords: Multicast, pragmatic general multicast, elastic reliable multicast, multicast-aware node, faulttolerance

\section{Introduction}

Multicasting has made group communication easier and cheaper. Examples include teleconferencing, video on demand, Internet TV, etc. The quality of multicasting can be enhanced if the fault-tolerance nature of the multicast protocols is studied and characterized such that its effect does not overwhelm and slow down the network. Multicast can be classified into two types, reliable and unreliable multicast. This paper focuses on reliable multicast such as the PGM and ERM, unreliable multicast is studied in another paper. Reliable multicast transport protocol are used by applications that required ordered, duplicatefree, multicast data delivery from multiple sources to multiple receivers. However, they can also be used in one-to-many group communication. 
Okonkwo O. Raphael Okonkwo, Akpojaro Jackson and Anthony Achuenu; Quantitative Analysis of the FaultTolerance of Pragmatic General Multicast (PGM) and Elastic Reliable Multicast (ERM) Protocols, Transactions on Networks and Communications, Volume 5 No. 2, April (2017); pp: 1-6

The advantages of group communication include [1]; less bandwidth is consumed for instance if a realtime feed of data packets from a source to various destinations is instantiated. As the number of destination increases less bandwidth is consumed while the reverse is the case with unicast model. Also, the server load (network load) is greatly reduced in a multicast model since the server has to send a packet once. Since only one packet is sent across the network, cost is greatly reduced compared to unicast model, which sends packets based on the number of receivers.

One of the salient advantages of reliable multicast protocols over traditional multicast protocols is that it guarantees that a receiver in the group either receives all data packets transmitted and retransmitted, or is able to detect unrecoverable data packet loss. Fault-tolerant computer systems are systems designed to be able to continue working to a certain level of satisfaction in the presence of faults. Therefore, the end to end delay and the amount of control bandwidth overhead (CBO) utilized when a fault occurs are studied and evaluated to provide useful information to the network community, particularly multicast designers, network integrators and users.

\section{Related Work}

The proliferation of group communication applications over the Internet has accelerated a stream of research in this field. Strigel and Manimaran [2] investigated the various issues and solutions for handling group dynamics and node failure in QoS-aware multicast models. In this work, the cost of maintaining dynamic multicast distribution trees with respect to changes in network topology as member join/leave the group was analysed and future research directions suggested.

In [3], the authors described E-Cast as a uniform causal-total-order multicast protocol designed to implement fault tolerant, highly elastic, yet strongly consistent database engines in the cloud. The work provides a rigorous formalization of routing problem, show how partial replication with strong consistency is guaranteed. Mir et al., evaluated the fault tolerance on protocol independent multicast (PIM) and core base tree (CBT) both of which are unreliable multicast protocols [4]. Performance evaluation parameters used included the end-to-end delay, network source usage and the overhead bandwidth cost. Their results showed that PIM performed better than CBT as the network size increases. Read carried out a multicast performance evaluation between PGM and multicast dissemination protocol with congestion control (MDP-CC) under different network conditions and concluded that PGM was superior to MDP-CC [5].

In [6], the authors investigated the technology which provides ubiquitous high bandwidth access for a large number of users in a wireless mesh network. The reliability of such network can significantly be degraded as broadcast traffic which is not solicited for by the participating nodes in the network. The work designed a self-pruning mechanism to control and reduce the broadcast traffic forwarding. The scheme defined two behaviours to manage the broadcasting operation while routing packets are managed differently from data broadcast messages to avoid afflicting the routing process. Simulated results show that the CBF ameliorates the network capacity by reducing considerably the number of redundant packets, thereby improving the end to end delay and providing high reachability and packet delivery.

In [7], the work surveyed multicast routing protocols with interest in ad-hoc networks. A general overview of multicast protocols and their performance was discussed. In particular, the work analysed the Ad-Hoc On-Demand Distance Vector (MAODV) [8], Adaptive Demand- Driven Multicast Routing protocol (ADMR) [9] and Core-Assisted Mesh Protocol (CAMP) [10]. It described how they work and showed reasons for 
developing these protocols. The work compared these protocols to explain their advantages and limitations.

An efficient approach for fault-tolerant capability for mobile multicast was presented in [11]. In multicast communication, packets can be concurrently sent from a source node to all members by multicast delivery tree. The main goal of the paper is that it makes multicast members immune from failure of nodes affection. The proposal contains two schemes. The first scheme uses the redundant resources of a mobile network to reconnect all the disconnected subtrees. The first scheme does not generate loops. In addition, it can control the maximum delivery delay of the new reconnected multicast delivery tree. The second scheme is initiated when the first scheme cannot reconnect all the subtrees. It extracts the failure-free part of the faulty multicast tree to form a safe multicast subtree. Then, multicast packets are only delivered along the safe multicast subtree to all the members. Unlike the first scheme, the second scheme is not based on the network topology support to achieve fault tolerance. Finally, simulations are performed to compare the proposed approach and previous approaches in terms of the fault-tolerant capability and various performance overheads.

\section{Methodology}

In this paper the end-to-end delay, the stress level of the source and the cost of control bandwidth overhead (CBO) for maintaining the multicast distribution trees were evaluated. The model is such that the source node floods the multicast routes with a control message and interested nodes indicate interest. A simulator program was designed to simulate the network. Faults were introduced randomly to signify dropped packets and the amount of $\mathrm{CBO}$ utilized were obtained for the two different protocols. The total amount of $\mathrm{CBO}$ utilized to repair broken distribution subtrees was calculated. The process was repeated for different network sizes.

A video stream from the source to the rendezvous point source (RPS) was sent by the RPS to connected receivers using the PRM and ERM protocols as shown in Figure 1. Faults were generated at random along some receivers (receivers are those with arrows) and the stress level at the RPS was computed based on the number of Negative Acknowledgement (NAK) and repair data that was sent between the RPS and the receivers.

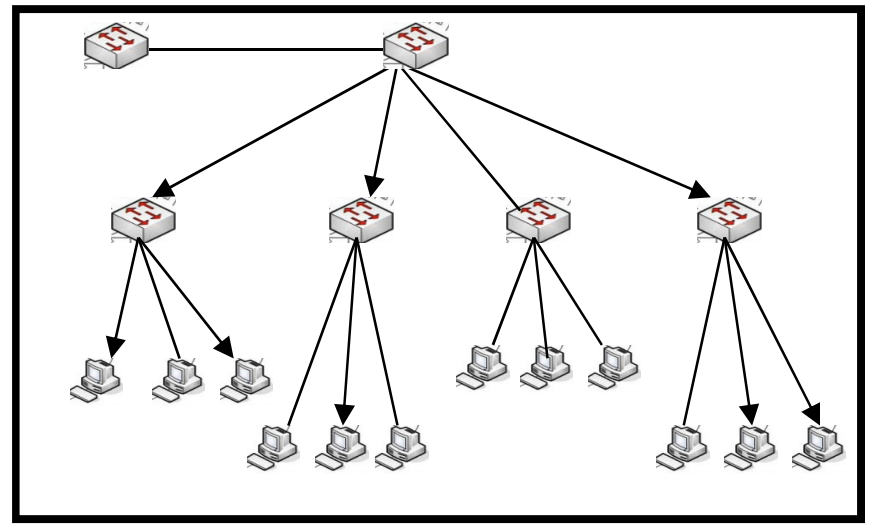

Figure 1: A hierarchical network showing multicast distribution tree 


\section{Analysis of Results}

Following from the above description, the stress level at the RPS for both PGM and ERM protocols were computed and the results presented in Table1. The receivers range was plotted against the stress level as shown in Figure 2. It is clearly shown that the stress level was more in the PGM protocol than in the ERM protocol. This shows that the ERM protocol more efficient than the PGM protocol since less CBO was used as the RPS performed less work in ERM protocol than in PGM protocol.

Table 1: Stress level at the RPS for PGM and ERM protocols

\begin{tabular}{|c|c|c|}
\hline $\begin{array}{c}\text { Range of } \\
\text { connected } \\
\text { leafs }\end{array}$ & $\begin{array}{c}\text { Stress } \\
\text { level for } \\
\text { PGM }\end{array}$ & $\begin{array}{c}\text { Stress } \\
\text { Level for } \\
\text { ERM }\end{array}$ \\
\hline $1-10$ & 46 & 22 \\
\hline $11-20$ & 203 & 135 \\
\hline $21-30$ & 323 & 274 \\
\hline $31-40$ & 495 & 397 \\
\hline $41-50$ & 634 & 499 \\
\hline Above 50 & 795 & 674 \\
\hline
\end{tabular}

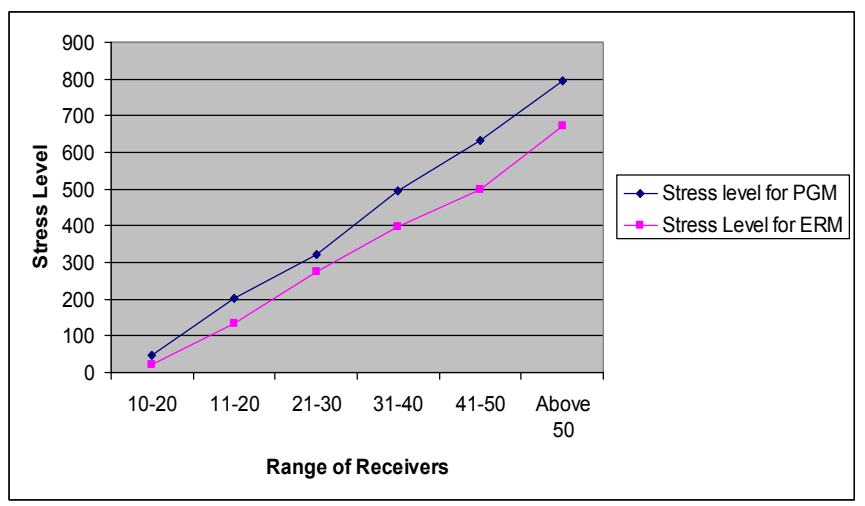

Figure 2: A graph showing the stress level at the RPS for PGM and ERM protocols.

Table 2 measures the cost of bandwidth overhead utilized at all level (source, RPS, studs and receivers' leaf routers). The size of receivers range was plotted against the overall control bandwidth overhead utilized as shown in Figure 3. The results show that the PGM protocol consistently consumed more CBO than the ERM protocol (Table 2). This implies that the distribution tree of the PGM protocol is more expensive to construct and maintain than that of the ERM distribution tree. 
Table 2: Comparison of CBO for PGM and ERM protocols

\begin{tabular}{|c|c|c|}
\hline $\begin{array}{c}\text { Range of } \\
\text { connected } \\
\text { leafs }\end{array}$ & CBO for PGM & $\begin{array}{c}\text { CBO for } \\
\text { ERM }\end{array}$ \\
\hline $1-10$ & 44 & 36 \\
\hline $11-20$ & 603 & 550 \\
\hline $21-30$ & 1666 & 1400 \\
\hline $31-40$ & 3249 & 2774 \\
\hline $41-50$ & 5568 & 4536 \\
\hline Above 50 & 8610 & 7370 \\
\hline
\end{tabular}

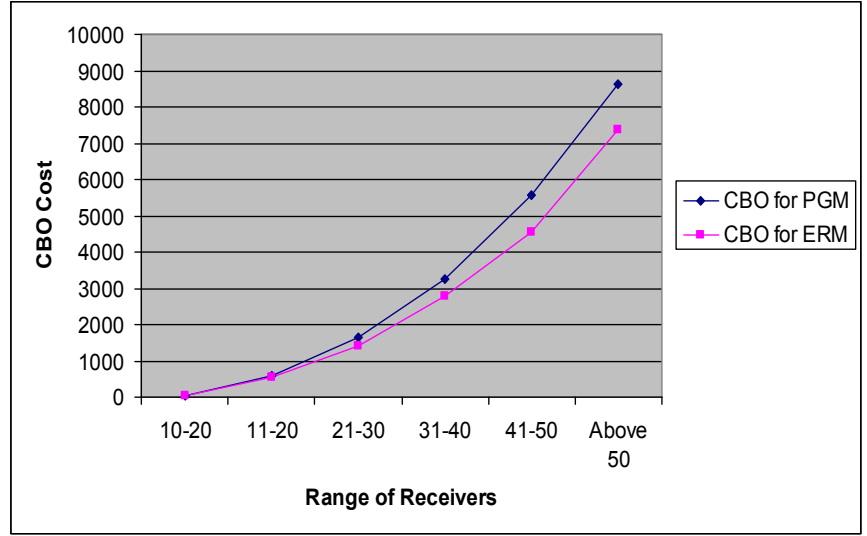

Figure 3: A graph showing the CBO utilized by both PGM and ERM protocols

\section{Conclusion}

This paper compares the fault-tolerance behaviour between PGM and ERM protocols using CBO utilized to construct and maintain their distribution trees. Findings show that the stress level at the RPS for ERM protocol is less than that of PGM protocol. This implies that the overall CBO for maintaining ERM protocol is less than that of PGM protocol. This means that the ERM protocol is more efficient than the PGM for the different sizes of multicast groups considered. This characterization is very important to the network community, particularly multicast designers, network integrators and users. This information is key while considering the improvement (or upgrade) of existing multicast protocols. The result is also significant at the early stage of designing new multicast protocols as it provides useful information in allocating scarce resource that can be appropriated to improve other infrastructure in the network.

\section{REFERENCES}

[1] Kaur, K, and Sachdeva, M., Performance matrices for evaluation of multicast routing. International Conference on Advances in Engineering, Science and Management (ICAESM), 2012, 582-587.

[2] Strigel, A., and Manimaran, G., Managing Group Dynamics and failures in QOS Multicasting. IEEE communications, 2002. 40(6): 82-87.

[3] Unterbrunner, P., Alonso, D., and Kossmann, G., E-Cast: Elastic Multicast. Technical Report, 2011. Available at https://www.researchgate.net/publication/275716927. 
Okonkwo O. Raphael Okonkwo, Akpojaro Jackson and Anthony Achuenu; Quantitative Analysis of the FaultTolerance of Pragmatic General Multicast (PGM) and Elastic Reliable Multicast (ERM) Protocols, Transactions on Networks and Communications, Volume 5 No. 2, April (2017); pp: 1-6

[4] Mir, N., Musa, S., Torresand, R., and Swamy, S., Evaluation of PIM and CBT Multicast Protocols on FaultTolerance. International Journal of Computing and Networking Technology, 2014. 2(2): 59-64.

[5] Read, N., A Multicast performance evaluation between PGM and MDP-CC under varying Network Conditions, 2006. Available at

https://vlebb.leeds.ac.uk/bbcswebdav/orgs/SCH Computing/FYProj/reports/0506/Read.pdf

[6] Youssef, S., Bouchaib, N., Soufiane, J., and Abdelkrim, H., Wireless Mesh Networks Capacity Improvement Using CBF. International Journal of Wireless \& Mobile Networks (IJWMN), 2015. 7(3): 1-15.

[7] Baker, R. M., and Akcayol, A. A. 2011. A Survey of Multicast Routing Protocols in Ad-Hoc Networks. Gazi University Journal of Science, GU J Sci., 2011. 24(3): 451-462.

[8] Royer, E. R., and Perkins, C. E., Multicast Operation of the Ad-hoc On-Demand Distance Vector Routing Protocol. In Proc. of the 5th annual ACM/IEEE International Conference on Mobile Computing and Networking (MobiCom), 1999. 207-218.

[9] Jetcheva, J. G., and Johnson, D. B., Adaptive Demand-Driven Multicast Routing in Multi-Hop Wireless Ad Hoc Networks. In Proceeding of the 2nd ACM International Symposium on Mobile and Ad-hoc Networking \& Computing, 2001. 33-44.

[10] Garcia-Luna-Aceves, J. J., and E. L. Madruga, E. L., The Core Assisted Mesh Protocol. IEEE Journal on Selected Areas in Communications, Special Issue on Ad-Hoc Networks, 1999. 17: 1380-1394.

[11] Lin, J., An Integrated Approach to Efficiently Providing Fault Tolerance for Mobile Multicast. Journal of Information Science and Engineering, 2005, 21: 153-179 